\title{
Reversible Cardiomyopathy Associated with Autoimmune Polyendocrine Syndrome Type II
}

\author{
Yusuf Karavelioglu ${ }^{1}$, Ahmet Baran ${ }^{2}$, Hekim Karapınar ${ }^{3}$, \\ Zekeriya Küçükdurmaz ${ }^{3}$ and Ahmet Yılmaz ${ }^{3}$
}

\begin{abstract}
Recovery of the ventricular function in a patient with cardiomyopathy is very rare. Autoimmune polyendocrine syndrome is also very rare. We herein report a case of reversed cardiomyopathy associated with autoimmune polyendocrine syndrome type II (Schmidt's syndrome) composed of Addison's disease, vitiligo and Hashimoto's thyroiditis. The ventricular function and size were reversed following the administration of suitable hormone replacement therapy for polyendocrine syndrome.
\end{abstract}

Key words: autoimmune polyendocrine syndrome II, adrenal insufficiency, hypothyroidism, vitiligo, cardiomyopathy, Takotsubo, cardiomyopathy

(Intern Med 52: 981-985, 2013)

(DOI: 10.2169/internalmedicine.52.7188)

\begin{abstract}
Introduction
Autoimmune polyendocrine syndrome type II (APS-II, Schmidt's syndrome) is a rare, immune-mediated endocrinopathy accompanied by autoimmune Addison's disease, autoimmune thyroid disease and/or type 1 diabetes mellitus (1). In many cases of autoimmune polyendocrine syndrome, autoimmune Addison's disease is associated with other autoimmune diseases, the most frequent being Hashimoto's thyroiditis. However, all three components (Addison's disease, thyroid disease and type 1 diabetes mellitus) are seen together in $10-20 \%$ of cases. Other rare components of the disease include additional autoimmune diseases such as vitiligo, alopecia, hypergonadotropic hypogonadism, chronic atrophic gastritis, chronic hepatitis and hypophysitis. Although it is not included in the definition criteria of the syndrome, cardiac involvement may be seen in rare cases (1). We herein report the case of a patient with APS-II that included Addison's disease, vitiligo and Hashimoto's thyroiditis who developed cardiomyopathy that recovered following the administration of appropriate endocrine therapy.
\end{abstract}

\begin{tabular}{l} 
Case Report \\
\hline A 36-year-old woman was admitted to our hospital with \\
fatigue, nausea and vomiting. She had experienced fatigue \\
for several months and nausea and vomiting for several \\
weeks. Her medical history revealed that she had suffered \\
from vitiligo for 20 years and had been followed up in a \\
psychiatry clinic for bipolar disorder. There were no overt \\
endocrine or cardiovascular diseases in her family history. \\
The patient was a non-smoker and non-alcohol consumer. \\
She had three living children and no menstrual irregularities. \\
She was cooperative and oriented but cachectic and ex- \\
hausted. She weighed 45 kilograms (kg) and was 165 centi- \\
meters (cm) in height. On inspection, extensive hyperpig- \\
mentation of the skin was noted with areas of vitiligo on the \\
hands, face and feet. No edema was observed and the pa- \\
tient's skin was dry. Her blood pressure was $90 / 60$ mmHg, \\
her heart rate was 60 beats per minute and rhythmic, her \\
respiratory rate was 13 breaths/minute and her SpO ${ }_{2}$ was \\
$98 \%$. Other system examinations were unremarkable. Chest \\
$\mathrm{X}$-ray revealed cardiac enlargement, clear pulmonary areas \\
and no pleural effusion. A sinus rhythm with T-wave nega- \\
tivity extended to the precordial and extremity leads was ob-
\end{tabular}

${ }^{1}$ Department of Cardiology, Çorum State Hospital, Turkey, ${ }^{2}$ Department of Internal Medicine, Çorum State Hospital, Turkey and ${ }^{3}$ Department of Cardiology, Cumhuriyet University, Medical School, Turkey

Received for publication December 25, 2011; Accepted for publication January 14, 2013

Correspondence to Dr. Hekim Karapınar, drhekim@yahoo.com 
Table. Laboratory Results at the Time of Admission and Follow-up

\begin{tabular}{|c|c|c|c|c|c|}
\hline & Baseline & $1^{\text {st }}$ month & $3^{\text {rd }}$ month & $1^{\text {st }}$ year & Normal values \\
\hline Glucose, mg/dL & 102 & 73 & 75 & 65 & $70-100$ \\
\hline $\mathrm{HbA} 1 \mathrm{c}, \mathrm{mg} / \mathrm{dL}$ & 5.33 & 4.9 & 5.6 & 5.4 & $4-6.1$ \\
\hline Urea, mg/dL & 45 & 42 & 39 & 40 & $0-50$ \\
\hline Creatinine, $\mathrm{mg} / \mathrm{dL}$ & 0.9 & 0.8 & 0.7 & 0.8 & $0.2-1.3$ \\
\hline Sodium, mmol/L & 127 & 135 & 135 & 139 & $134-148$ \\
\hline Potassium, mmol/L & 7.5 & 3.7 & 3.8 & 4.2 & $3.5-5.5$ \\
\hline Chloride $\mathrm{mmol} / \mathrm{L}$ & 104 & 101 & 97 & 100 & $90-108$ \\
\hline Calcium, mg/dL & 7.6 & 8.2 & 8.2 & 8.3 & $8-11$ \\
\hline Phosphor, mg/dL & 3.5 & - & - & 4.1 & $2.5-4.5$ \\
\hline Thyroid-stimulating hormone, $\mathrm{mIU} / \mathrm{L}$ & 31 & 3.6 & 3.1 & 2.3 & $0.4-4.2$ \\
\hline Free triiodothyronine, $\mathrm{pg} / \mathrm{mL}$ & 3.73 & 4.18 & 4.09 & 3.62 & $2.3-4.2$ \\
\hline Free thyroxine, ng/dL & 1.08 & 1.50 & 1.45 & 1.53 & $0.72-1.56$ \\
\hline Alanine transaminase, $\mathrm{U} / \mathrm{L}$ & 34 & 25 & 24 & 22 & $0-50$ \\
\hline Aspartate transaminase, $\mathrm{U} / \mathrm{L}$ & 16 & 15 & 20 & 21 & $0-50$ \\
\hline Creatine kinase, $\mathrm{U} / \mathrm{L}$ & 65 & 70 & 73 & 71 & $25-170$ \\
\hline Creatine kinase-MB fraction, $\mathrm{U} / \mathrm{L}$ & 20 & 15 & 16 & 18 & $5-25$ \\
\hline Lactate Dehydrogenase IU/L & 357 & 330 & 300 & 310 & $240-480$ \\
\hline Troponin $\mathrm{T}, \mu \mathrm{g} / \mathrm{L}$ & $<0.01$ & - & - & $<0.01$ & $<0.01$ \\
\hline Cortisol, $\mu \mathrm{g} / \mathrm{dL}$ & $<1$ & 1.1 & 3.1 & 3 & $5.0-25.0$ \\
\hline Adrenocorticotropic hormone, $\mathrm{pg} / \mathrm{mL}$ & $>1,250$ & 38 & 41 & 33 & $10-52$ \\
\hline Hemoglobin $\mathrm{g} / \mathrm{dL}$ & 10.6 & 10.9 & 11.4 & 12.4 & $11.5-16.5$ \\
\hline Hematocrit, \% & 35.1 & 36.4 & 38.5 & 41 & $37-47$ \\
\hline Parathormone, $\mathrm{pg} / \mathrm{mL}$ & 76.5 & - & - & - & $10-70$ \\
\hline Prolactin, ng/mL & 12.06 & - & - & - & $3.5-20$ \\
\hline Follicle-stimulating hormone, $\mathrm{mIU} / \mathrm{mL}$ & 20.2 & - & - & - & $6-26$ \\
\hline Luteinizing hormone, $\mathrm{mIU} / \mathrm{mL}$ & 17 & - & - & - & $20-75$ \\
\hline Estradiol, $\mathrm{pg} / \mathrm{mL}$ & 98 & - & - & - & $19-140$ \\
\hline Vitamin B12, pg/mL & 362 & - & - & - & $191-663$ \\
\hline Folate, $\mathrm{ng} / \mathrm{mL}$ & 11.7 & - & - & - & $3-17.5$ \\
\hline Albumin, $\mathrm{g} / \mathrm{dL}$ & 3.9 & - & - & - & $3.5-5.0$ \\
\hline Anti-thyroid peroxidase antibody, $\mathrm{U} / \mathrm{mL}$ & 942 & - & - & - & $0-30$ \\
\hline Anti-thyroglobulin antibody, $\mathrm{U} / \mathrm{mL}$ & 37 & - & - & - & $<20$ \\
\hline
\end{tabular}

served on electrocardiography (ECG).

Biochemical analyses revealed hyponatremia (124 meq/L) and hyperkalemia $(7.5 \mathrm{meq} / \mathrm{L})$. The serum urea and creatinine levels were normal (Table). The serum troponin I level was also normal and the level of N-terminal probrain natriuretic peptide (NT-proBNP) was $135 \mathrm{pg} / \mathrm{mL}$. Because the elevated potassium level did not decrease despite the administration of medical therapy, the patient was admitted to the intensive care unit where she underwent dialysis for a single session. The potassium level returned to the normal range after dialysis. The patient had also fatigue despite normal electrolyte levels. A blood sample analysis showed a low serum cortisol level $(<1 \mu \mathrm{g} / \mathrm{dL}$, normal range: 5-25 $\mu \mathrm{g}$ / $\mathrm{dL}$, at 8 a.m.) and an elevated adrenocorticotropic hormone level $(1,250 \mathrm{pg} / \mathrm{mL}$, normal range: $10-52 \mathrm{pg} / \mathrm{mL})$. Dynamic magnetic resonance imaging (MRI) of the pituitary gland was normal. MRI of the abdomen, including the adrenal glands, was normal. Serology for human immunodeficiency virus and the hepatitis $\mathrm{B}$ and $\mathrm{C}$ viruses was normal. A puri- fied protein derivative (PPD) tuberculin skin test was nonreactive. The patient was diagnosed with Addison's disease based on her clinical and laboratory findings.

The thyroid-stimulating hormone (TSH) level was elevated (31 mIU/L, normal range: 0.4-4.2 mIU/L), the free triiodothyronine and free thyroxin levels were in the normal ranges and the anti-thyroid peroxidase antibody titer was high $(942 \mathrm{U} / \mathrm{mL})$. Thyroid gland ultrasound showed a normal-sized, heterogeneous thyroid gland without nodules. The ultrasound findings were compatible with a diagnosis of Hashimoto's thyroiditis, which was made based on these findings.

Taking the presence of vitiligo, Addison's disease and Hashimoto's thyroiditis into consideration, the patient was diagnosed with APS II and evaluated for other components of APS type II. The presence of type 1 diabetes mellitus was assessed according to the plasma fasting glucose and glycolized hemoglobin 1c (HbA1c) levels and glucose tolerance tests; however, the results did not support a type 1 dia- 


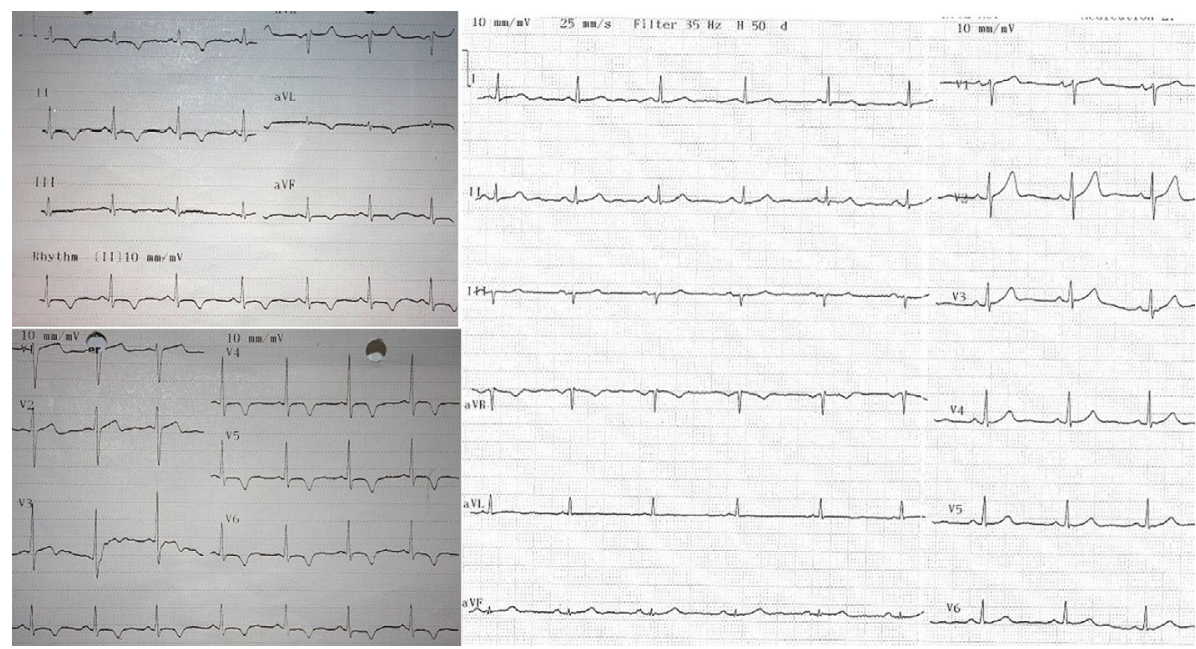

Figure 1. Electrocardiogram obtained at the time of hospital admission shows negative T-waves extending to the precordial and extremity leads (A). Electrocardiogram performed at the third month is completely normal after the administration of appropriate treatment $(B)$.
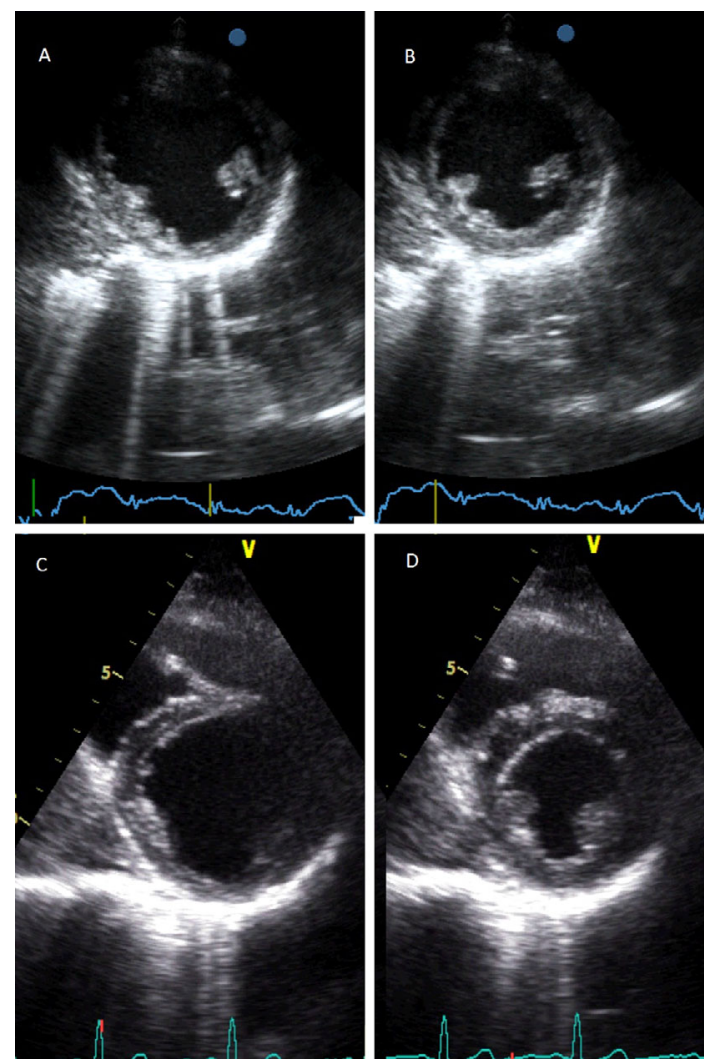

Figure 2. Transthoracic echocardiogram obtained at the time of hospital admission shows increased diastolic $(5.9 \mathrm{~cm}$, normal $<5.1 \mathrm{~cm})(\mathrm{A})$ and systolic $(5.0 \mathrm{~cm}$, normal $<3.8 \mathrm{~cm})(\mathrm{B})$ dimensions and a decreased ejection fraction $(30 \%$, normal $>50 \%$ ) using Simpson's methods. Transthoracic echocardiogram obtained at the third month after hospital admission shows normal diastolic $(4.9 \mathrm{~cm})(\mathrm{C})$ and systolic $(3.3 \mathrm{~cm})(\mathrm{D})$ dimensions and a normal ejection fraction (62\%) using Simpson's methods.

betes diagnosis.

A cardiovascular examination was performed to assess cardiac involvement. Control ECG performed after dialysis revealed a sinus rhythm with persistent T-wave negativity on the precordial and extremity leads (ECG, Fig. 1A). Echocardiography revealed an enlarged left ventricle with a depressed systolic function, akinesis of the anterior, apical and septal segments and hypokinesis of the other segments (Fig. 2A, B) with mild mitral and tricuspid regurgitation. The estimated left ventricular end diastolic pressure was slightly elevated $\left(E / e^{\prime}=12\right)$. Mild spontaneous echo contrast was noted in the left ventricular cavity.

The patient was administered prednisolone at a dose of $7.5 \mathrm{mg}$, fludrocortisone at a dose of $0.1 \mathrm{mg}$ and levothyroxine at a dose of $50 \mathrm{mcg}$ daily due to the high TSH levels and clinical findings of hypothyroidism such as depression. Her complaints and findings began to resolve after the first week. On the 1st month follow-up visit, the patient's complaints were alleviated, and the New York Heart Association functional capacity improved from class II to class I. On the 3rd month follow-up visit, the ECG and echocardiography findings were found to be completely recovered (Figs. 1B, 2C, D), while the estimated left ventricular end diastolic pressure $\left(\mathrm{E} / \mathrm{e}^{\prime}=6\right)$ and NT-proBNP levels $(95 \mathrm{pg} /$ $\mathrm{mL}$ ) were normal. While under daily maintenance therapy with $5 \mathrm{mg}$ of prednisolone, $100 \mathrm{mcg}$ of levothyroxine and $0.1 \mathrm{mg}$ of fludrocortisone at the 3rd month follow-up visit, the patient's blood pressure was $115 / 75 \mathrm{mmHg}$, her heart rate was 63 beats per minute and her blood levels of serum sodium, potassium, glucose, cortisol, adrenocorticotropic hormone and thyroid-stimulating hormone were normal. Her body weight had increased from $45 \mathrm{~kg}$ to $55 \mathrm{~kg}$ at the $3 \mathrm{rd}$ month follow-up visit and to $65 \mathrm{~kg}$ at the 1 st year follow-up visit. The patient was completely asymptomatic while under the maintenance therapy. The baseline and follow-up biochemical and hormonal analysis results of the blood samples are shown in Table. 


\section{Discussion}

We herein presented a case of APS-II with cardiomyopathy that completely recovered with appropriate treatment. In most cases of APS-II, autoimmune Addison's disease is associated with Hashimoto's thyroiditis, whereas three components (Addison's disease, thyroid disease and type 1 diabetes mellitus) are seen together in $10-20 \%$ of cases. According to recent epidemiological data, the frequency of APS-II is estimated to be 4-5 cases per 100,000 individuals in the general population (2). Other autoimmune diseases such as vitiligo, alopecia, hypergonadotropic hypogonadism, chronic atrophic gastritis, chronic hepatitis and hypophysitis are minor components that are likely to accompany the syndrome. The present patient had Addison's disease, Hashimoto's thyroiditis and vitiligo.

Left ventricular dysfunction was observed in the present case. This has rarely been reported in the literature and is not included in the definition or classification criteria of APS (1). Myocardial systolic dysfunction has been reported to be reversible in most cases reported in the literature. Left ventricular systolic dysfunction was reported to manifest as apical ballooning (Takotsubo cardiomyopathy, TC) in one case (3) in which the patient was observed to have STsegment elevation and elevated levels of myocardial necrosis markers at the time of hospital admission, although the ventricular function recovered after a few days, as in other apical ballooning cases. TC is characterized by acute onset and quickly reversible left ventricular apical wall motion abnormalities with chest symptoms, ST-segment elevation and Twave inversion on ECG, minimal-moderate myocardial enzymatic release and no significant stenosis on coronary angiography (CAG). Ukita et al. reported another case of a patient with acute adrenal insufficiency and TC (4). We did not consider our patient to have TC, although the cardiac involvement was similar, because TC typically exhibits an acute course and occurs in postmenopausal women. However, in our case, the patient's clinical course was chronic and she was in a reproductive period. In addition, TC presents with mild-moderate cardiac enzyme elevation; however, these markers were normal in our patient. Furthermore, the cardiac function recovers over a short time period (days to a couple of weeks) in patients with TC, while our patient's cardiac function recovered in weeks to months. Finally, in this case, recovery was observed only after the administration of appropriate hormone replacement therapy and did not occur spontaneously, as in patients with TC. The previously reported case is unlike the recent case with respect to the acute clinical course. However, the effects of chronic stress (e.g., adrenal insufficiency) on the cardiac function are unknown and should be evaluated in prospective studies.

Another patient with APS-II presented with heart failure after delivery and was diagnosed with peripartum cardiomyopathy (5). The authors reported that the patient recov- ered at the first month follow-up after the administration of hormone replacement treatment (5). Unfortunately, another reported patient with worsened heart failure was referred for cardiac transplantation (6). In addition, pulmonary hypertension was observed in an APS-II case reported by Saliba et al. (7), and right ventricular dysfunction was observed in an isolated adrenocorticotropic hormone deficiency case reported by Shimizu et al. (8). Therefore, APS-II not only may be associated with left ventricular systolic dysfunction, but also may exhibit myocardial and vascular involvement. In the present case, the patient's pulmonary blood pressure calculated via tricuspid regurgitation was normal. There were no signs of right ventricular involvement.

Apart from APS-II, the development of left ventricular dysfunction in patients with pure adrenocorticotropic hormone deficiency (8-10) might originate from adrenal gland dysfunction. The contribution of electrolyte imbalances, which were noted at the time of hospital admission in the present case, to the development of transient left ventricular dysfunction may be a possible factor. Severe hyponatremia increases the intracellular calcium concentrations by inducing sodium and calcium ion channel dysfunction in the cell membrane, which might be reflected as myocardial dysfunction in the clinical picture. The fact that the hyponatremia observed in the present case was not very severe and returned to a normal level in the early term reduces this possibility. Glucocorticoids play important roles in myocardial contraction. Impaired calcium uptake in the sarcoplasmic reticulum and decreased microsomal phosphorylase activity in adrenalectomized rats are associated with myocardial dysfunction $(11,12)$. Furthermore, it has also been reported that intracellular calcium concentrations are affected by glucocorticoid hormones that bind to cardiac ion channels (13). Therefore, we suggest that the reversible ventricular wall motion abnormalities noted in the present case might have resulted from alterations in intracellular calcium concentrations due to hyponatremia and adrenal insufficiency.

APS is a rare endocrine syndrome. Cardiac signs have been reported, although rarely. While the causes of APS have not yet been clearly established, cardiac involvement presents with systolic dysfunction of the myocardium rather than dysfunction in the conduction system. For patients with APS who are evaluated to have diagnoses of cardiac failure or myocardial infarction, it is beneficial to keep this reversible picture in mind. Speculations on its etiology will become clearer with the identification of more cases.

\section{The authors state that they have no Conflict of Interest (COI).}

\section{References}

1. Neufeld M, Blizzard RM. Polyglandular autoimmune disease. In: Symposium on Autoimmune Aspects of Endocrine Disorders. Pinchera A, Doniach D, Fenzi FG, Baschieri L, Eds. Academic Press, New York, 1980: 357-365.

2. Laureti S, Vecchi L, Santeusanio F, Falorni A. Is the prevalence of 
Addison's disease underestimated? J Clin Endocrinol Metab 84: 1762, 1999

3. Lim T, Murakami H, Hayashi K, et al. Takotsubo cardiomyopathy associated with autoimmune polyendocrine syndrome II. J Cardiol 53: 306-310, 2009.

4. Ukita C, Miyazaki H, Toyoda N, Kosaki A, Nishikawa M, Iwasaka T. Takotsubo cardiomyopathy during acute adrenal crisis due to isolated adrenocorticotropin deficiency. Intern Med 48: 347-352, 2009.

5. Kumar KV, Pushkaraj SG, Krishnaleela BL, Modi KD. Peripartum cardiomyopathy in type II autoimmune polyendocrine syndrome. Int J Cardiol 149: e14-e15, 2011.

6. Nielsen TD, Steenbergen C, Russell SD. Nonischemic cardiomyopathy associated with autoimmune polyglandular syndrome type II. Endocr Pract 13: 59-62, 2007.

7. Saliba WR, Rock W, Elias M. Pulmonary hypertension in a patient with Schmidt syndrome. Am J Emerg Med 27: 1025.e11025.e2, 2009.
8. Shimizu M, Monguchi T, Takano T, Miwa Y. Isolated ACTH deficiency presenting with severe myocardial dysfunction. J Cardiol Cases 4: e26-e30, 2011.

9. Afzal A, Khaja f. Reversible cardiomyopathy associated with Addison's disease. Can J Cardiol 16: 377-379, 2000.

10. Conwell LS, Gray LW, Delbridge RG, Thomsett MJ, Batch JA. Reversible cardiomyopathy in pediatric Addison's disease: a cautionary tale. J Pediatr Endocrinol Metab 16: 1191-1195, 2003.

11. Narayanan $\mathrm{N}$. Effects of adrenalectomy and in vivo administration of dexamethasone on ATP-dependent calcium accumulation by sarcoplasmic reticulum from rat heart. J Mol Cell Cardiol 15: 715, 1983.

12. Rao MK, Xu A, Narayanan N. Glucocorticoid modulation of protein phosphorylation and sarcoplasmic reticulum function in rat myocardium. Am J Physiol Heart Circ Physiol 281: H325-H333, 2001.

13. Penefsky ZJ, McCann FV. Electrical properties of developing rat heart. Effects of dexamethasone. J Dev Physiol 12: 27-33, 1989.

(C) 2013 The Japanese Society of Internal Medicine http://www.naika.or.jp/imonline/index.html 